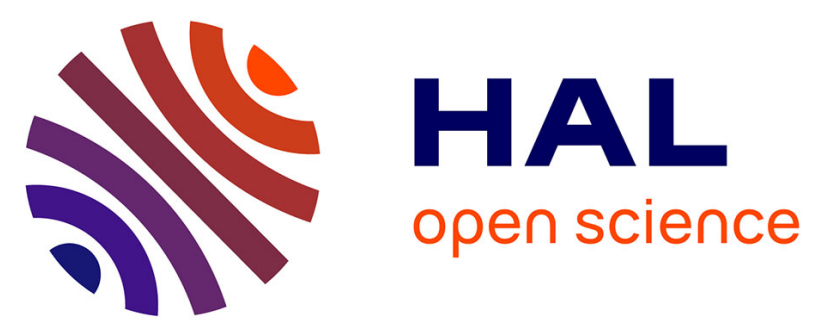

\title{
Textures of cholesteric droplets controlled by photo-switching chirality at the molecular level
} Jiawen Chen, Emmanuelle Lacaze, Etienne Brasselet, Syuzanna R. Harutyunyan, Nathalie Katsonis, Ben L. Feringa

\section{- To cite this version:}

Jiawen Chen, Emmanuelle Lacaze, Etienne Brasselet, Syuzanna R. Harutyunyan, Nathalie Katsonis, et al.. Textures of cholesteric droplets controlled by photo-switching chirality at the molecular level. Journal of Materials Chemistry C, 2014, 2 (38), pp.8137-8141. 10.1039/C4TC01297F . hal-01068137

\section{HAL Id: hal-01068137 \\ https://hal.science/hal-01068137}

Submitted on 19 Feb 2016

HAL is a multi-disciplinary open access archive for the deposit and dissemination of scientific research documents, whether they are published or not. The documents may come from teaching and research institutions in France or abroad, or from public or private research centers.
L'archive ouverte pluridisciplinaire HAL, est destinée au dépôt et à la diffusion de documents scientifiques de niveau recherche, publiés ou non, émanant des établissements d'enseignement et de recherche français ou étrangers, des laboratoires publics ou privés.

\section{다(1)(2)}

Distributed under a Creative Commons Attribution - ShareAlikel 4.0 International 


\title{
Textures of cholesteric droplets controlled by photo-switching chirality at the molecular level $\dagger$
}

\author{
Jiawen Chen, ${ }^{a}$ Emmanuelle Lacaze, ${ }^{\text {b }}$ Etienne Brasselet, ${ }^{c}$ Syuzanna R. Harutyunyan, ${ }^{a}$ \\ Nathalie Katsonis ${ }^{* d}$ and Ben L. Feringa*a \\ We demonstrate the ultra-sensitive detection of molecular chirality experimentally, by investigating \\ structural transitions in sessile droplets of cholesteric liquid crystals. By doping the liquid crystal with \\ chiral molecular photo-switches, we demonstrate that both optical and structural transitions can be \\ triggered by light, reversibly. Moreover, we show that depending on the initial confinement state of the \\ cholesteric helix, the expression of chirality can be photo-switched on and off by means of an interplay \\ between microscopic and geometric constraints.
}

\section{Introduction}

Liquid crystals hold potential for the development of smart materials because they respond to a variety of external stimuli such as temperature, pressure, electric fields, chemical contamination and light. ${ }^{1}$ Moreover, changes of molecular organization in thin films of liquid crystals can be monitored optically because they are expressed at the macroscopic level of the liquid crystal texture, which is of special interest for sensing. Efforts to harness the stimuli-responsiveness of liquid crystal films include optical monitoring of bio-molecular interactions, ${ }^{2}$ detection of gases, ${ }^{3}$ and the detection of chiral molecules in an adjacent gaseous phase. ${ }^{4}$ Thin films of liquid crystals are also used in displays and their ability to control the amplitude, phase and polarization of light is at the heart of many applications in the field of photonics. ${ }^{5}$ Besides their optical properties, we have shown that photo-responsive films of cholesteric liquid crystals undergo a continuous rotation under irradiation, which can be harnessed to produce work. ${ }^{6,7}$

Recent developments suggest that the impact and applicability of approaches based on the confinement of liquid crystals in thin films could be substantially enhanced by confining the liquid crystals into the third dimension. Indeed, droplets of liquid crystals have been found to be far more sensitive to ordering transitions than planar films. ${ }^{8}$ Another recent example

${ }^{a}$ Stratingh Institute for Chemistry, University of Groningen, Nijenborgh 4, 9747 AG Groningen, The Netherlands. E mail: b.l.feringa@rug.nl

${ }^{b}$ CNRS UMR7588, Institut des Nano Sciences de Paris (INSP), Paris, France

${ }^{c}$ Laboratoire Ondes et Matière d'Aquitaine, CNRS, Université Bordeaux 1, 351 Cours de la Libération, $F 33400$ Talence, France

${ }^{d}$ MESA+ Institute for Nanotechnology, University of Twente, 7500 AE Enschede, The Netherlands.E mail:n.h.katsonis@utwente.nl has revealed that tactoidal sessile droplets of liquid crystals are of fundamental interest for understanding chiral symmetry breaking. ${ }^{9}$ In photonics, the use of spherical droplets has provided new opportunities such as optical vortex generation ${ }^{\mathbf{1 0}}$ and photonic cross-communication. ${ }^{11}$ Moreover, combining the droplet geometry with the orientational sensitivity of liquid crystals to electric fields has led to the realization of electrically tuneable lasers from achiral droplets of nematic liquid crystals. $^{12}$

Manipulating the chiral orientational features of liquid crystal droplets consequently appears as an issue of fundamental and practical interest. Electrical control over the handedness of chiral features has been demonstrated for spherical droplets of nematic liquid crystals with perpendicular ${ }^{13}$ or tangential $^{14}$ anchoring boundary conditions. The control over chiral features can also be achieved by using electromagnetic fields, as demonstrated in radial droplets that were optically twisted using circularly polarized tweezers. ${ }^{15}$ In contrast to these approaches, the use of light as an external stimulus allows controlling chirality from the molecular to the mesoscopic and eventually the macroscopic level. ${ }^{16}$ Still, while molecular-driven control over chirality has been demonstrated in thin films, ${ }^{6,7}$ in situ and continuous variation in the texture of liquid crystal droplets remains to be demonstrated and understood. Here we demonstrate photo-control over the structures formed by cholesteric liquid crystals in sessile droplets. We demonstrate that optically rewritable chiral textures can be created and controlled, whether the initial droplet displays a frustrated geometry or not.

\section{Results and discussion}

The cholesteric liquid crystals used in this study are prepared by doping the nematic liquid crystal E7 with a few percent of chiral dopant 1, whose concentration determines the pitch $p$. Sessile 
droplets with a typical diameter $D=20-30 \mu \mathrm{m}$ and height $h$ are obtained by mechanical shear strain on silanized glass that imposes a local alignment which is perpendicular to the surface (homeotropic alignment). These droplets having prepared in air, the liquid crystal director also experiences perpendicular boundary conditions at the cholesteric/air interface. As illustrated in Fig. 1b, all the droplets have a spherical cap shape whose contact angle with the substrate is constant independent of the droplet. Invariance in the shape of the droplets ensures that the ratio $D / h$ is constant and found to be $\approx 3.1$ (Fig. $1 \mathrm{~b}$ ). Even so, the texture of a cholesteric droplet strongly depends on the droplet height and pitch, as illustrated in Fig. 2, where droplets with $h / p=0.4$ (Fig. 2a) and $h / p=7.4$ (Fig. 2b) are observed between crossed polarizers. This feature is similar to the case of spherical droplets where the parameter controlling the texture of the droplet is $R / p$, where $R$ is the radius of the droplet. The controlling nature of the ratio $R / p$ in determining the texture of spherical cholesteric droplets was demonstrated for both tangential and normal anchoring. ${ }^{17,18}$

In fact, the ratio $h / p$ turns out to be a relevant parameter for classification of the textures which are observed before irradiation, which we from now on refer to as the 'orientational ground state'. The importance of this ratio can be better understood by comparison with cholesteric films subjected to homeotropic boundary conditions. In these films, the spatial confinement parameter given by the ratio $L / p$, where $L$ is the film thickness, dictates the geometry of the orientational ground state. In the present case, the height $h$ of the sessile droplet plays the role of $L$ for films. Since the intrinsic helical order is incompatible with the orientational boundary conditions, frustration takes place. ${ }^{19}$ As a consequence, for droplets whose size is small compared to the pitch, the director field at rest can be unwound whereas for larger droplets molecular chirality is expressed up to the macroscopic level. Quantitatively, the critical value for the unwinding-winding transition in films is $L / p=K_{3} /\left(2 K_{2}\right),{ }^{20}$ where $K_{2}$ and $K_{3}$ are the twist and bend Frank elastic constants of the liquid crystal. Based on the abovementioned analogy we found for the present nematic host E7, for which $K_{2}=7.4 \mathrm{pN}$ and $K_{3}=16.5 \mathrm{pN}$ at $25^{\circ} \mathrm{C}$ temperature, ${ }^{21} \mathrm{a}$ critical height to pitch ratio would be $h / p \approx 1$.Therefore, we expect that the cholesteric liquid crystal is unwound in droplets a)

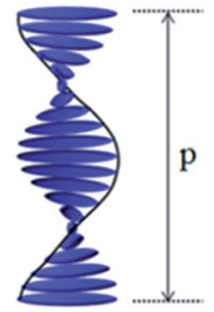

b)

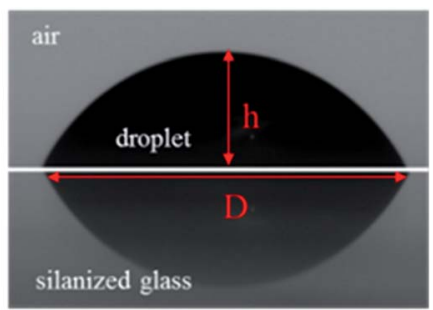

Fig. 1 (a) Schematic representation of a cholesteric liquid crystal. Each rod represents the local average molecular orientation of the liquid crystal (the director). The director rotates by $2 \pi$ over a distance that defines the cholesteric pitch $p$. (b) Side view of a sessile droplet of the cholesteric liquid crystal deposited on a silanized glass and surrounded by air.

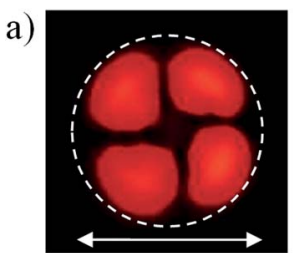

$D=22 \mu \mathrm{m}$

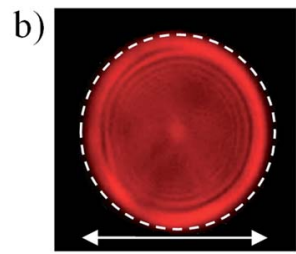

$D=30 \mu \mathrm{m}$

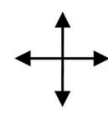

Fig. 2 Transmission intensity pattern of sessile cholesteric liquid crystal droplets observed between crossed polarizers. Dashed circles refer to the area of the droplet on the substrate and double arrows indicate the direction of the polarizers. A $560 \mathrm{~nm}$ cut-off filter was used in order to increase the contrast of the textures. The focus was made close to the upper face of the substrate. (a) Tilted cross-pattern ( $p \quad 17 \mu \mathrm{m}, D \quad 22 \mu \mathrm{m}, h / p \quad$ 0.4). The cholesteric mixture used to create these droplets contains $\sim 0.1 \mathrm{wt} \%$ of dopant (mixture a). (b) Onion-like pattern ( $p \quad 1.3 \mu \mathrm{m}, D \quad 30 \mu \mathrm{m}, h / p \quad$ 7.4). The cholesteric mixture used to create these droplets contains $\sim 1 \mathrm{wt} \%$ of dopant (mixture b).

with $h / p=0.4$ (Fig. 2a) whereas it is wound in droplets characterized by a confinement ratio $h / p=7.4$ (Fig. 2b). This allows understanding the qualitatively different optical transmission patterns that are observed between crossed polarizers (Fig. 2).

Our first observation is that the dark cross-pattern observed for the unwound droplet is similar to the cross-pattern observed for a pure (achiral) nematic liquid crystal (not shown here), though it is left-tilted in the present case (Fig. 2a). Two phenomena could be at the origin of such a tilt: either the orientational ordering of the director is chiral, or the chirality of the dopants is expressed through their optical activity. In the former case, the polarization plane of light would adiabatically follow the director's orientation, which means that the tilt originates from chirality at the supramolecular level whereas in the latter case, the tilt would originate from chirality at the molecular level. Here, we note that the relatively low concentration of chiral dopants in the mixtures used (ranging from 0.1 $\mathrm{wt} \%$ to $1 \mathrm{wt} \%$ ) is not expected to substantially modify the elastic constants of the nematic host. Moreover, given that between crossed polarizers, the orientational ground state observed for pure nematic droplets is a straight dark cross-pattern, we estimate that the emergence of chirality by formation of a chiral supramolecular arrangement is not likely to occur here. Thus, we conclude that the observed tilt is a direct consequence of microscopic chirality, namely the optical activity of the chiral dopant.

We also observed that wound droplets exhibit an onion-like transmission pattern between crossed polarizers that is nearly invariant by rotation and radially modulated (Fig. 2b). This indicates that linearly polarized light emerges from the droplet in a polarization state that depends on the radial coordinate of the droplet. By analogy with the fingerprint structure observed for thin films at the silanized-glass-air interface, ${ }^{7}$ such an observation could be explained by the formation of quasicircular stripes due to the confined geometry of the sessile droplet. An alternative explanation could be related to the existence of a radially dependent axisymmetric optical 
retardation profile. This would be reminiscent of the wellknown onion structure in spherical droplets although we are not yet able to suggest a chiral three-dimensional director field that would be relevant for sessile droplets. Either way, we found that, in contrast to the unwound situation, a chiral supramolecular organization is at work within the droplet.

Independent from the orientational ground state of a cholesteric droplet, one can expect to be able to modify either its 3D organization and/or its optical response by modifying the helical twisting power of the chiral dopant, while the concentration in chiral dopant remains constant. Here, this externally controlled and reversible behaviour is achieved by using a photosensitive cholesteric liquid crystal. This cholesteric mixture is prepared by doping a nematic host with an achiral dopant whose structure can be modified by irradiation..$^{22,23}$ Overcrowded alkenes are molecular switches displaying a unique efficiency in modifying the pitch and handedness of the cholesteric helix ${ }^{24}$ that has been used to photo-control cholesteric textures in thin films ${ }^{6}$ and in optical cells. ${ }^{25}$ Overcrowded alkene 1 (Fig. 3) constitutes an efficient dopant for a large variety of nematic liquid crystals owing to its high helical twisting power both in its stable form 1-(P) and in its photostationary state 1-(M). Importantly, the stable form 1-(P) and its isomer 1-(M) induce helices of opposite handedness, as expressed in the opposite signs of their HTP (Fig. 3). Using these photo-active dopants allows tuning chirality at will by controlling the UV dose delivered to the material. In a practical way, for a given mixture of photoactive cholesteric, the duration of irradiation in order to reach a predetermined value of the cholesteric pitch depends on the light intensity and wavelength. In the present case, the absorption spectrum of overcrowded alkene 1 indicates that the cholesteric helix can be activated by irradiation at $\lambda=365 \mathrm{~nm}$, a wavelength that also provides an optimal value in the photostationary state. ${ }^{26}$

For droplets displaying an initial spatial confinement ratio of $h / p=0.4$, transmission of light between crossed polarizers is shown in the stable state (Fig. 4a), and upon constant

a)

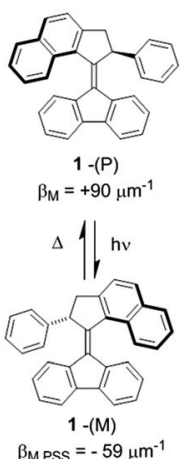

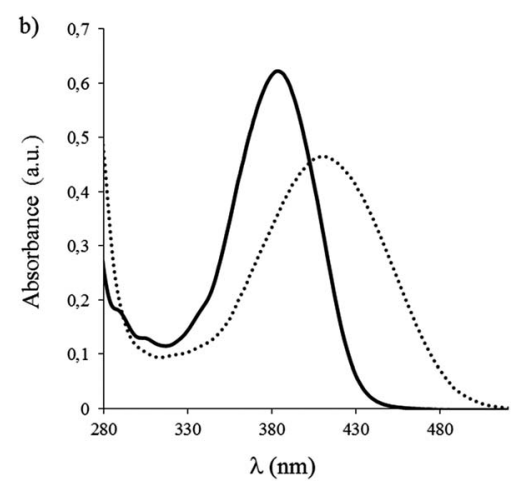

Fig. 3 Photo-controlled manipulation of chirality in sessile droplets of cholesteric liquid crystals. (a) Photochemical isomerization from 1-(P) to 1-(M) and thermal helix inversion from 1-(M) back to 1-(P). $\beta_{M}$ refers to the helical twisting power in E7, in the stable state and in the photostationary state (PSS). (b) UV/vis spectra of 1-(P) and 1-(M) in hexane (solid line and dotted line, respectively).

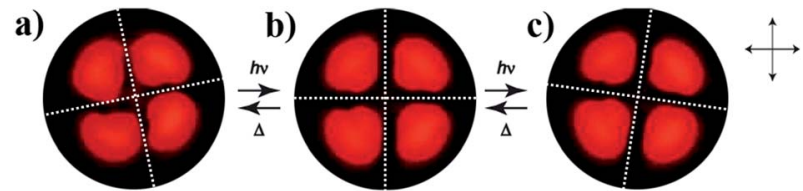

Fig. 4 Photo-controlled modification of optical activity in a droplet where the cholesteric helix is initially frustrated $(h / p \quad 0.4)$. From left to right, irradiation leads to a continuous change of the tilt angle of the dark cross. The process is fully reversible thermally.

irradiation with $\lambda=365 \mathrm{~nm}$, as the exposure time increases (Fig. 4b and c). As described above, a tilted extinction cross is observed in the stable state. Upon irradiation with UV light, the axis of the extinction cross undergoes a clockwise rotation that reflects the increasing concentration of 1-(M) that gradually cancels out the contribution of 1-(P) in the resulting helical twisting power, which therefore decreases. In turn, the cholesteric pitch increases until the overall optical activity of the droplets vanishes and the mixture becomes 'pseudo-nematic' (Fig. 4b). We refer to this state as pseudo-nematic because the pseudo-racemic mixture contains chiral isomers having opposite helical twisting powers, which means that the resulting effect is equivalent to a racemic mixture where the effects of chirality cancel out. As we proceed with UV irradiation, chirality is recovered in the optical signature of the droplet, but with an opposite handedness (Fig. 4c). Reversion of the tilt angle of the cross indicates that it is the 1-(M) species that dictates the sign of the overall helical twisting power. Importantly, at any stage of the irradiation process, the light-induced modifications can be stopped and reversed by turning off UV irradiation. The process is completed after a few minutes and its exact kinetics depends on the intensity of irradiation. Consequently, we have demonstrated that the optical signature of droplets displaying geometric frustration can be controlled reversibly by using light. All chiral textures observed can be understood without involving modifications in the distribution of the director within the droplet.

In droplets where no frustration occurs, and where the helix is initially wound, the switching of textures involves a reorganization of the cholesteric liquid crystal at the supramolecular level. Fig. 5 shows the photo-induced structural and optical transitions in a cholesteric droplet where no geometric frustration is observed before irradiation with UV light $(h / p=7.4)$. The corresponding movie is available in the ESI (Movie $1 \dagger$ ). Transmission between crossed polarizers is displayed in the stable state (Fig. 5a), when a pseudo-racemic mixture of 1-(P) and 1-(M) is formed (Fig. 5c), and in the photostationary state (Fig. 5e). As before, these states are obtained successively as the irradiation time increases. The initial onion-like texture disappears progressively: the concentric rings separate from each other, until they fade away. Photo-induced increase of the distance between two successive rings agrees with a gradual unwinding of the cholesteric helix as the pitch increases. While the concentric rings are expanding, a dark extinction area is formed and spreads abruptly over the whole droplet to give rise to an optical transmission pattern in which a tilted extinction 

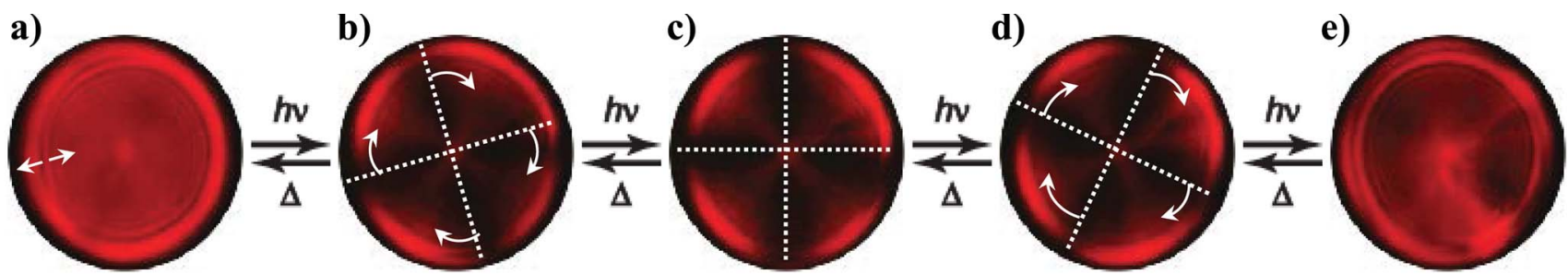

Fig. 5 Photo-induced structural and optical transitions in a cholesteric droplet where no geometric frustration is observed prior to irradiation ( $h$ / $p$ 7.4). a) Onion-like texture at $t \quad 0$. b) Tilted cross texture (t $47 \mathrm{~s}$ ). c) Straight cross texture (t 54 s). d) Tilted cross texture, with opposite chirality than observed in $5 b$ (t 66 s). e) Onion-like texture (t 100 s). Both structural and optical transitions are fully reversible.

cross dominates (Fig. 5b). We suggest that this structural transition originates in the continuous increase of the cholesteric pitch that is eventually expected to reach a threshold. Past this threshold, spatial confinement induces a structural transition through reorientation of the director. Structural transitions have been observed experimentally ${ }^{27}$ and described theoretically $^{28}$ in tangentially anchored nematic droplets. Other structural transitions induced by spatial confinement have been observed in tactoidal droplets also. ${ }^{9}$

Despite prolonged irradiation, it is surprising to note that the concentric rings characteristic of the onion-like feature do not disappear completely after the structural transition has taken place. A few rings are still visible at the periphery of the drop - i.e. near the interface with air. We attribute these residual features to constraints in geometry related to homeotropic boundary conditions, which work against unwinding of the helix at the proximity of the interface. It is reasonable to assume that in these areas, the volume effects are unable to overcome the surface effects.

After the structural transition occurs, the tilt direction indicates that the 1-(P) species dictates the sign of the overall helical twisting power (Fig. 5b). Upon further irradiation, a pseudoracemic mixture of 1-(P) and 1-(M) is formed as the tilt of the cross decreases and eventually disappears (Fig. 5c). As irradiation continues, the texture rotates and the optical signature of chirality is recovered (Fig. 5d), but in the opposite direction, which is the signature of an opposite helical handedness. After the frustration threshold is reached, the concentric rings become clearer and narrower, and the extinction area disappears (Fig. 5e). The texture of the droplet in the photostationary state is similar to the texture observed initially, which is in agreement with similar values of helical twisting power for 1-(P) and 1-(M), and with the high conversion rate of the photoreaction. This texture is chiral both at the molecular and at the supramolecular level of the helix, but its chirality is not expressed in its optical signature. All steps are fully reversible thermally.

\section{Experimental}

E7 is a eutectic mixture which is nematic at room temperature and was supplied by Merck.

All chemical reagents used for the synthesis of molecular motor 1 were purchased from Sigma-Aldrich. Motor 1 was synthesized following the procedures reported in ref. 29.
Enantioresolution of 1 was performed using chiral stationary phase HPLC: Chiralpak OD column, $98: 2 n$-heptane : 2-prop-

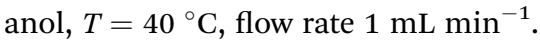

A Spectroline pencil lamp was used for irradiation at $\lambda=365$ $\mathrm{nm}$, with a nominal intensity of $1 \mathrm{~mW} \mathrm{\textrm {cm } ^ { - 2 }}$ at $2.5 \mathrm{~cm}$.

\section{Conclusions}

In conclusion, we have extended the study of photo-responsive thin films to sessile droplets of cholesteric liquid crystals, and confirmed the role of geometric frustration in the expression of chirality at the macroscopic level. After investigating the interplay between the surface and bulk forces in these droplets, we conclude that perpendicular anchoring enhances the optical appearance of the structural transitions, resulting in a clear tilt of extinction bands and the formation of onion-like textures. These results suggest that structural transitions in sessile cholesteric droplets hold promise towards the ultra-sensitive detection of molecular chirality.

\section{Acknowledgements}

This work was supported financially by a Vidi grant from the Netherlands Organization for Scientific Research (NK), by an ERC Starting Grant $(307784$, NK) and by an ERC Advanced Investigator Grant (227897, BLF).

\section{Notes and references}

1 P. G. de Gennes, The Physics of Liquid Crystals, Oxford University Press, Oxford, 1974.

2 J. M. Brake, M. K. Daschner, Y.-Y. Luk and N. L. Abbott, Science, 2003, 302, 2094.

3 Y. Han, K. Pacheco, C. W. M. Bastiaansen, D. J. Broer and R. P. Sijbesma, J. Am. Chem. Soc., 2010, 132, 2961.

4 T. Ohzono, T. Yamamoto and J. Fukuda, Nat. Commun., 2014, 5, 3735.

5 H. Coles and S. Morris, Nat. Photonics, 2010, 4, 676.

6 R. Eelkema, M. M. Pollard, J. Vicario, N. Katsonis, B. S. Ramon, C. W. M. Bastiaansen, D. J. Broer and B. L. Feringa, Nature, 2006, 440, 163.

7 A. Bosco, M. G. M. Jongejan, R. Eelkema, N. Katsonis, E. Lacaze, A. Ferrarini and B. L. Feringa, J. Am. Chem. Soc., 2008, 130, 14615. 
8 H. Lin, D. S. Miller, P. J. Bertics, C. J. Murphy, J. J. de Pablo and N. L. Abbott, Science, 2011, 332, 1297.

9 L. Tortora and O. D. Lavrentovich, Proc. Natl. Acad. Sci. U. S. A., 2011, 108, 5163.

10 E. Brasselet, N. Murazawa, H. Mizawa and S. Juodkazis, Phys. Rev. Lett., 2009, 103, 103903.

11 J. Noh, H.-L. Liang, I. Drevensek-Olenik and J. P. F. Lagerwall, J. Mater. Chem. C, 2014, 2, 806.

12 M. Humar, M. Ravnik, S. Pajk and I. Musevic, Nat. Photonics, 2009, 3, 597.

13 F. Xu, H. S. Kitzerow and P. P. Crooker, Phys. Rev. A, 1992, 46, 6535.

14 P. Rudquist, E. Korblova, D. M. Walba, R. Shao, N. A. Clark and J. E. Maclennan, Liq. Cryst., 1999, 26, 1555.

15 (a) N. Murazawa, S. Juodkazis, S. Matuso and H. Misawa, Small, 2005, 1, 656; (b) E. Brasselet, N. Murazawa, S. Juodkazis and H. Misawa, Phys. Rev. E: Stat. Phys., Plasmas, Fluids, Relat. Interdiscip. Top., 2008, 77, 041704.

16 T. Ikeda, J. Mater. Chem., 2003, 13, 2037.

17 M. Kurik and O. D. Lavrentovich, JETP Lett., 1982, 35, 444.

18 F. Xu and P. P. Crooker, Phys. Rev. E: Stat. Phys., Plasmas, Fluids, Relat. Interdiscip. Top., 1997, 56, 6853.
19 R. D. Kamien and J. V. Selinger, J. Phys.: Condens. Matter, 2001, 13, R1.

20 B. Y. Zeldovich and N. V. Tabiryan, JETP Lett., 1981, 34, 406408.

21 J. F. Stromer, Appl. Phys. Lett., 2006, 88, 051915.

22 R. Eelkema, Liq. Cryst., 2011, 38, 1641.

23 N. Katsonis, E. Lacaze and A. Ferrarini, J. Mater. Chem., 2012, 22, 7088.

24 M. M. Pollard, M. Klok, D. Pijper and B. L. Feringa, Adv. Funct. Mater., 2007, 17, 718.

25 T. J. White, S. A. Cazzell, A. S. Freer, D.-K. Yang, L. Sukhomlinova, L. Su, T. Kosa, B. Taheri and T. J. Bunning, Adv. Mater., 2011, 23, 1389.

26 J. Vicario, A. Meetsma and B. L. Feringa, Chem. Commun., 2005, 5910.

27 G. E. Volovik and O. D. Lavrentovich, JETP Lett., 1983, 58, 1159.

28 R. D. Williams, J. Phys. A: Math. Gen., 1986, 19, 3211.

29 J. Vicario, M. Walko, A. Meetsma and B. L. Feringa, J. Am. Chem. Soc., 2006, 128, 5127. 